\title{
Dendritic to Globular Morphology Transition in Ternary Alloy Solidification
}

\author{
Denis Danilov and Britta Nestler \\ Institute of Applied Research, Karlsruhe University of Applied Sciences, Moltkestrasse 30, 76133 Karlsruhe, Germany
}

(Received 3 June 2004; published 15 November 2004)

\begin{abstract}
The evolution of solidification microstructures in ternary metallic alloys is investigated by adaptive finite element simulations of a general multicomponent phase-field model. A morphological transition from dendritic to globular growth is found by varying the alloy composition at a fixed undercooling. The dependence of the growth velocity and of the impurity segregation in the solid phase on the composition is analyzed and indicates a smooth type of transition between the dendritic and globular growth structures.
\end{abstract}

DOI: 10.1103/PhysRevLett.93.215501

PACS numbers: 81.10.Aj, 61.66.Dk, 68.70.+w, 81.30.Fb

Multicomponent alloys form the most important class of metallic materials for technical and industrial processes. Combined with the number of components is a wealth of different phases, solidification processes, and pattern formations. The mechanical properties of a material strongly depend on the morphology and on the characteristical quantities of the microstructure. One of the most common growth morphologies in metallic alloys are dendrites. During growth, dendritic patterns generate solute microsegregation forming the structure of grain boundaries on a larger scale. The growth of dendrites from an initially undercooled metallic melt is caused by a negative concentration gradient ahead of the solidification front. Extensive studies have been made to explore dendritic growth in pure substances and in binary alloys. The constitutional undercooling and the corresponding concentration gradient at the growing front are the main factors leading to morphological instabilities of the solid-liquid interface [1,2]. In a binary alloy, the relation between the temperature interval $\Delta T_{0}$ of the liquidus and solidus line, the concentration of the impurity component $c_{0}$ in the melt, the partition coefficient $k_{e}$, and the liquidus slope $m_{e}$ is given by $\Delta T_{0}=-m_{e} c_{0}(1-$ $\left.k_{e}\right) / k_{e}$, according to the equilibrium phase diagram of a stationary planar front. The temperature interval determines the magnitude of the constitutional undercooling at a growing solid-liquid interface and is hence a significant material quantity for the stability properties. The more pronounced concentration boundary layer of the impurity ahead of the moving interface at larger values $\Delta T_{0}$ drives the occurrence of instabilities leading to the formation of cellular and/or dendritic structures. For multicomponent alloys as, e.g., in ternary systems, the interface instabilities are driven by the characteristic diffusion properties of each solute field in the multicomponent system. Using an approximated model with independently acting solutes, it was analytically shown in [3] that each solute enhances morphological instability of a solidification front with different weighting factors. A variation of the alloy composition causes a change of the strength of instability forces on the growing interface, because the value of the liquidus-solidus temperature interval strongly depends on the alloy composition. Investigations of the interacting quantities and of the fundamental mechanisms during solidification of ternary or higher component materials have just recently become more intensive by means of numerical modeling.

To numerically treat the complex solid-liquid interface geometry of dendritic crystals, a phase-field approach has the advantage of avoiding the explicit tracking of the phase boundary. The solid and liquid are distinguished by a phase-field variable $\phi(x, t)$ with a smooth transition from 0 to 1 leading to a diffuse interface. In the sharp interface limit, classical free boundary problems and the Gibbs-Thomson law can be recovered from the phasefield approach (see, e.g., [4], and references therein). In the two past decades, there has been a great progress in phasefield modeling of growth structures in pure substances and alloy systems. For a historical background and applications, see the review article in [5], and references therein.

In this Letter, the influence of changing the alloy composition on the interface stability, on the characteristic morphology, and on the growth velocity is investigated by performing numerical simulations of a general multicomponent phase-field model with interacting and coupled diffusion fields. We chose the ternary $\mathrm{Ni}_{60} \mathrm{Cu}_{40-x} \mathrm{Cr}$ alloy system as a prototype for this study and herewith build upon intensive studies of the binary $\mathrm{Ni}-\mathrm{Cu}$ system (e.g., [6,7]). Hence, the corresponding physical parameters are established relatively well. Furthermore, the liquidus-solidus interval for binary $\mathrm{Ni}_{60} \mathrm{Cu}_{40}$ is about 3 times larger than the interval for binary $\mathrm{Ni}_{60} \mathrm{Cr}_{40}$ and one can expect a significant dependence of growth morphology on the concentration of the alloy components.

For the subsequent computations of ternary alloy solidification, we apply a recently developed phase-field model to the case of three components and two phases. The general formulation of the phase-field model in [8] 
allows for an arbitrary number of phases and components and is based on an entropy density functional of the form

$$
S=\int_{\Omega}\left\{s(e, c, \phi)-\left[\varepsilon a(\phi, \nabla \phi)+\frac{1}{\varepsilon} w(\phi)\right]\right\} d x .
$$

The bulk entropy density $s$ depends on the internal energy $e$, on a concentration vector $c=\left(c_{1}, \ldots, c_{N}\right)$, and on a phase-field vector $\phi=\left(\phi_{1}, \ldots, \phi_{M}\right)$. The vector components $c_{i}, i=1, \ldots, N$, represent the solute concentrations in an $N$-component alloy. The variable $\phi_{\alpha}, \alpha=1, \ldots, M$, denotes the local volume fraction of phase $\alpha$ in an $M$-phase system. The thermodynamics of the interfaces is determined by the gradient energy density $a(\phi, \nabla \phi)$, by the multiwell potential $w(\phi)$, and by the small scale parameter $\varepsilon$ related to the thickness of the interface. The gradient energy and the multiwell potential depend on the surface entropy density $\gamma$. The evolution equations are obtained from Eq. (1) in a thermodynamically consistent way ensuring a combination of positive local entropy production and mass conservation laws. By variational derivatives $\partial_{t} \phi_{\alpha} \sim \frac{\delta S}{\delta \phi_{\alpha}}-\lambda$, the phase-field equations read

$$
\omega \varepsilon \frac{\partial \phi_{\alpha}}{\partial t}=\varepsilon\left(\nabla \frac{\partial a}{\partial \nabla \phi_{\alpha}}-\frac{\partial a}{\partial \phi_{\alpha}}\right)-\frac{1}{\varepsilon} \frac{\partial w}{\partial \phi_{\alpha}}-\frac{1}{T} \frac{\partial f}{\partial \phi_{\alpha}}-\lambda,
$$

where $f(c, T, \phi)$ is the free energy density, $\lambda$ is a Lagrange multiplier taking the constraint $\phi_{1}+\cdots+$ $\phi_{M}=1$ for the phase fields into account, and $\omega$ is a kinetic factor related to the kinetic coefficient $\mu$. In the sharp interface limit, it has been discussed in [8] that Eq. (2) relates to the Gibbs-Thomson equation for a moving interface.

In our numerical investigations, the alloy composition will be changed by keeping the $\mathrm{Ni}$ concentration at a constant 60 at. \% and by exchanging $\mathrm{Cu}$ by $\mathrm{Cr}$, i.e., $\mathrm{Ni}_{60} \mathrm{Cu}_{40-x} \mathrm{Cr}_{x}$ with $0<x<40$ at. $\%$. Three assumptions are applied: (i) We examine primary dendritic growth involving only two phases in the ternary system: a solid and a liquid. The eutectic region in the $\mathrm{Ni}-\mathrm{Cr}$ phase diagram is not considered. For a solid-liquid system, the phase-field model reduces to one variable $\phi$ denoting the fraction of solid phase. (ii) The system is considered in ideal solution approximation with a free energy density of the form

$$
f(c, \phi)=\sum_{i=1}^{3} c_{i} L_{i} \frac{T-T_{i}}{T_{i}} h(\phi)+\frac{R T}{v_{m}} \sum_{i=1}^{3} c_{i} \ln c_{i},
$$

where $L_{i}$ and $T_{i}$ are the latent heat and the melting temperature of component $i$, respectively, $R$ is the gas constant, $v_{m}$ is the molar volume, and $h(\phi)$ is a monotone function on $[0,1]$ that satisfies $h(0)=0$ and $h(1)=1$. (iii) Following [6], we impose an isothermal temperature field $T$. This approximation is valid because the thermal diffusivity of the alloy is about 4 orders of magnitude larger than the solutal diffusivity. In addition, for the considered undercooling, the growth takes place under diffusion limited conditions.

Surface energy and kinetic anisotropy are incorporated into the model by formulating orientation dependent gradient energy $a(\phi, \nabla \phi)$ and kinetic factor $\omega(\phi, \nabla \phi)$ :

$$
\begin{gathered}
a(\phi, \nabla \phi)=\gamma|\nabla \phi|^{2}=\gamma_{0}\left[1+\epsilon_{c} \cos (4 \theta)\right]|\nabla \phi|^{2}, \\
\omega(\phi, \nabla \phi)=\omega_{0}\left[1-\epsilon_{k} \cos (4 \theta)\right],
\end{gathered}
$$

where $\theta$ is the angle between the vector $\nabla \phi$ and the $x$ axis.

Assuming the mass fluxes to be linear functions of the thermodynamic driving forces $\mu_{i}=\frac{\partial f}{\partial c_{i}}$, the mass balance equations for the three components are

$$
\frac{\partial c_{i}}{\partial t}=-\nabla\left[\sum_{j=1}^{3} L_{i j}(c, \phi) \nabla \frac{-\mu_{j}}{T}\right],
$$

with mobility coefficients given by

$$
L_{i j}(c, \phi)=\frac{v_{m}}{R} D_{i} c_{i}\left(\delta_{i j}-\frac{D_{j} c_{j}}{\sum_{k=1}^{3} D_{k} c_{k}}\right) .
$$

The form of Eq. (7) allows different values of the bare trace diffusion coefficients $D_{i}(\phi)$ for the different components $i$ and satisfies the constraint $c_{1}+c_{2}+c_{3}=1$.

Numerical aspects of phase-field modeling have been discussed in [9] including the conditions for spatial grid resolution in comparison to the interface thickness. The evolution Eqs. (2) and (6) are solved using a finite element method with a semi-implicit time discretization on a nonuniform adaptive mesh having the highest order of spatial resolution in the vicinity of the solid-liquid interface where the gradients of the phase field and of the concentrations reach maximal values. The adaptive grid refinement criterion has been defined ensuring a minimum of 7-10 grid points in regions of the diffuse phase boundary. To verify the origin of interface instability, simulations with different time and spatial resolution have been performed leading to the same growth morphology. Hence, it has been shown that the interfacial instabilities are of Mullins-Sekerka-type and not numerically induced.

The solidus and liquidus lines of the binary $\mathrm{Ni}-\mathrm{Cu}$ and of the $\mathrm{Ni}-\mathrm{Cr}$ phase diagram can be constructed from the free energy, Eq. (3), using the melting temperatures $T_{\mathrm{Ni}}=1728 \mathrm{~K}, T_{\mathrm{Cu}}=1358 \mathrm{~K}, T_{\mathrm{Cr}}=1465 \mathrm{~K}$, the latent heats $L_{\mathrm{Ni}}=2350 \mathrm{~J} / \mathrm{cm}^{3}, \quad L_{\mathrm{Cu}}=1728 \mathrm{~J} / \mathrm{cm}^{3}$, $L_{\mathrm{Cr}}=1493 \mathrm{~J} / \mathrm{cm}^{3}$, and the molar volume $v_{m}=$ $7.42 \mathrm{~cm}^{3}$. The values of the melting temperature and of the latent heat for $\mathrm{Cr}$ are adjustable parameters in order to recover the actual binary phase diagram in the given region of concentrations. The above values lead to a partition coefficient $k_{e}=0.843$, to a liquidus slope $m_{e}=$ $-3.27 \mathrm{~K} /$ at. $\%$, and to the undercooling $\Delta T_{0}=24.4 \mathrm{~K}$ 
for the binary $\mathrm{Ni}_{60} \mathrm{Cu}_{40}$ system. Similarly, we obtain $k_{e}=$ $0.905, m_{e}=-2.08 \mathrm{~K} /$ at. $\%$, and $\Delta T_{0}=8.7 \mathrm{~K}$ for the binary $\mathrm{Ni}_{60} \mathrm{Cr}_{40}$ system on the corresponding equilibrium phase diagram.

To accentuate the effect of solidification interval, we assume that surface properties (surface energy density $\sigma$ and kinetic coefficient $\mu$ ) do not depend on alloy composition and have the values $\sigma=\gamma_{0} T=0.37 \mathrm{~J} / \mathrm{m}^{2}$ and $\mu=3.3 \mathrm{~mm} /(\mathrm{s} \mathrm{K})[6]$. The anisotropy of the interface properties plays an important role in the selection of the operating state during dendritic growth. In this study, we use the values obtained from molecular-dynamics simulations for the anisotropies in pure Ni, Eqs. (4) and (5), $\epsilon_{c}=0.023$ for surface free energy density, and $\epsilon_{k}=$ 0.169 for the kinetic coefficient [7]. As reported in [7], the diffusion coefficients of $\mathrm{Ni}$ and $\mathrm{Cu}$ in melt are $D_{\mathrm{Ni}}=$ $3.82 \times 10^{-9} \mathrm{~m}^{2} / \mathrm{s}, D_{\mathrm{Cu}}=3.32 \times 10^{-9} \mathrm{~m}^{2} / \mathrm{s}$, and we assume $D_{\mathrm{Cr}}=1.5 \times 10^{-9} \mathrm{~m}^{2} / \mathrm{s}$. The diffusion coefficient in the solid phase is set equal to $10^{-13} \mathrm{~m}^{2} / \mathrm{s}$ for all components. The value of the small length scale parameter in the entropy functional, Eqs. (1) and (2), is chosen to be $\varepsilon=0.1 \mu \mathrm{m}$.

We conducted numerical computations for different alloy compositions varying from $\mathrm{Ni}_{60} \mathrm{Cu}_{36} \mathrm{Cr}_{4}$ to $\mathrm{Ni}_{60} \mathrm{Cu}_{4} \mathrm{Cr}_{36}$ and for fixed undercooling conditions. We kept the concentration of $\mathrm{Ni}$ at 60 at. \% and adjusted the initial undercooling at $20 \mathrm{~K}$ measured from the equilibrium liquidus line for a given composition. The corresponding shapes of growing crystals are shown in Fig. 1. At small contents of $\mathrm{Cr}$, the solid phase forms a dendritic structure that has a pronounced preferable growth direction determined by the anisotropy of surface energy and interface kinetics (first three images of Fig. 1). An increase of $\mathrm{Cr}$ concentration in the melt leads to an increase of dendritic tip velocity $V$. This is accompanied by a thickening of the primary dendritic trunk. Further rise of Cr concentration (middle three images of Fig. 1) causes a morphological transition from a dendritic to a globular growth shape at a composition $\mathrm{Ni}_{60} \mathrm{Cu}_{20} \mathrm{Cr}_{20}$. The globular form of the crystals is stable after this morphological transition for further change of alloy composition to $\mathrm{Ni}_{60} \mathrm{Cu}_{4} \mathrm{Cr}_{36}$. Within the globular regime of solidification, the anisotropy of the interface has a smaller effect on the crystal shape. The preferred growth directions are less pronounced in comparison with the dendritic shape for amounts of $\mathrm{Cr}$ less than 20 at. \%. To investigate the dependence of the spatial redistribution of the alloy components in the solid phase on the melt composition, we consider isolines corresponding to the average concentration of nickel atoms in the solid phase. These isolines separate Ni depleted and Ni enriched domains (see Fig. 1, solid lines). The character of the isolines for copper and

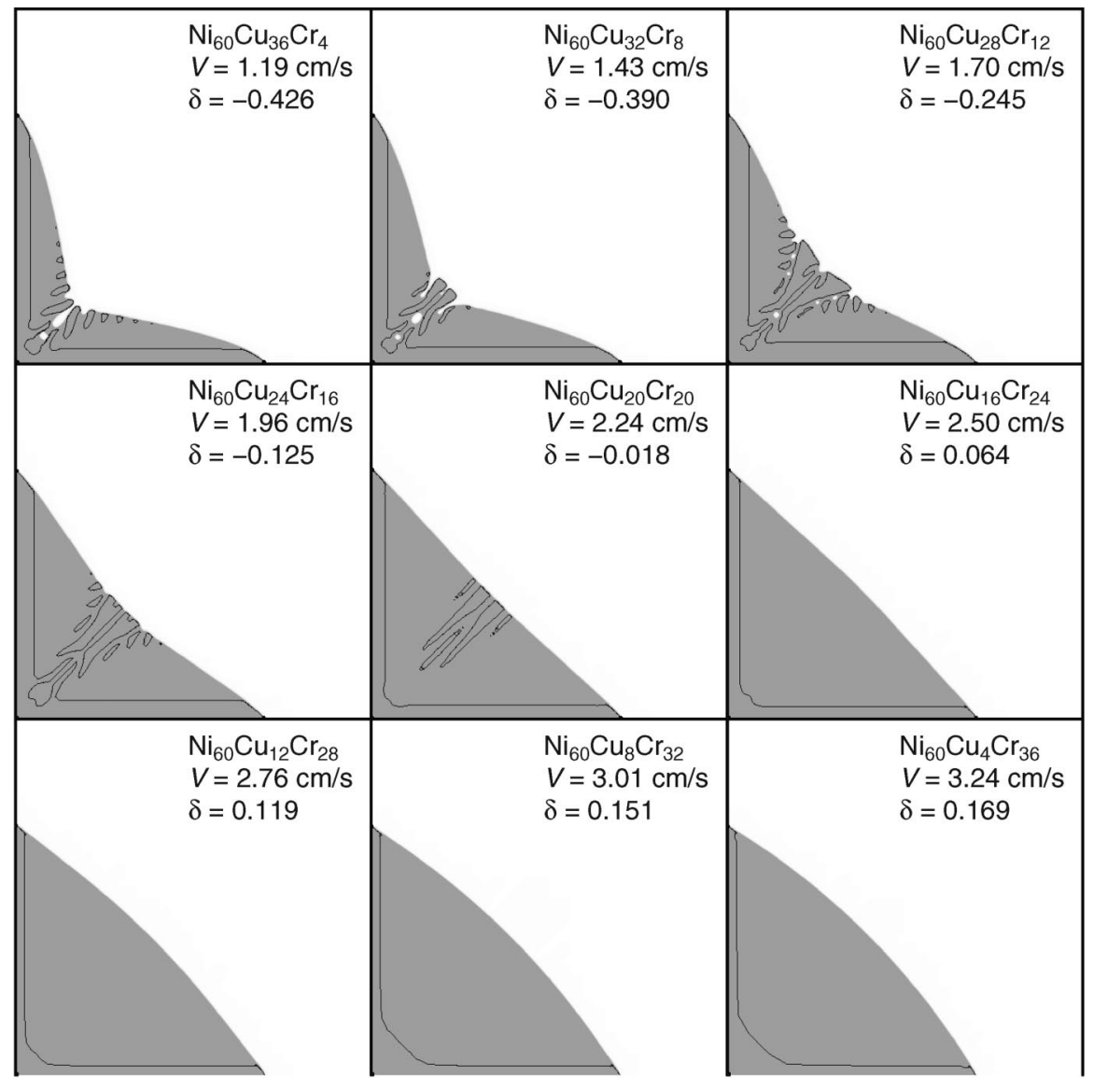

FIG. 1. Transition from dendritic to globular morphology for different initial melt compositions at a constant initial undercooling of $20 \mathrm{~K}$. Shaded regions correspond to the solid phase, i.e., $\phi \geq 0.5$, and solid lines represent the isolines of average concentration of $\mathrm{Ni}$ in solid. The values for the tip velocities $V$ and for the area estimations $\delta$ are stated in each image. 


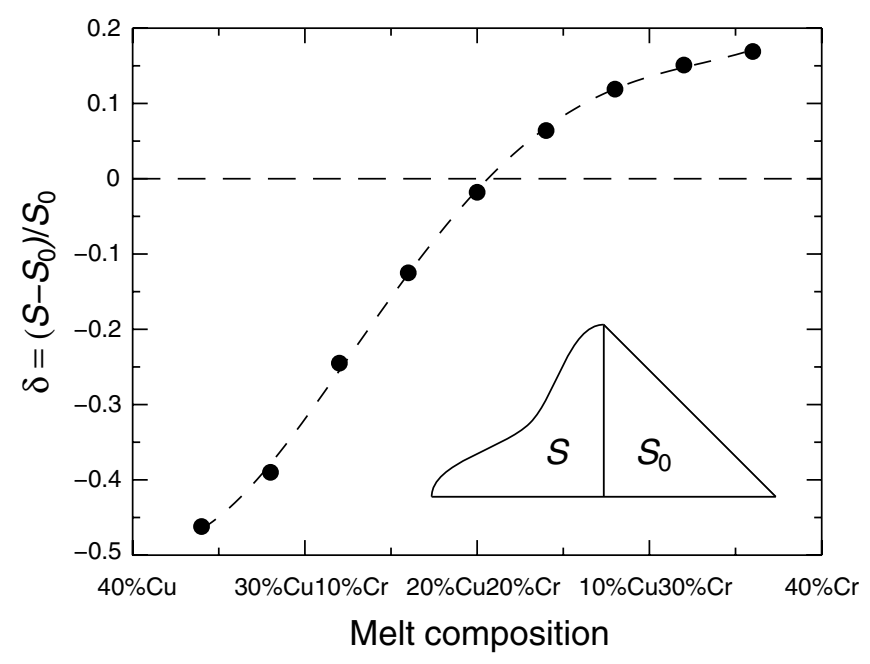

FIG. 2. Simulated area $S$ covered by the crystal in relation to a normalized triangular area $S_{0}$ for different melt compositions. The $\delta=0$ line marks the transition from dendritic to globular growth morphologies.

chrome is qualitatively the same having only small quantitative deviations. Two independent parts can be found in the geometry of the isolines. The first part is aligned in a direction parallel to the dendritic trunk remaining in this position after the transition to the globular morphology. The second part develops in diagonal direction and it disappears at the morphological transition.

The morphological transition can be measured quantitatively using the deviation $\delta=\left(S-S_{0}\right) / S_{0}$ of area $S$ covered by the solid phase from the triangular area $S_{0}$ between the dendritic tips and the center of the initial seed, Fig. 2. With the change of melt composition, the deviation $\delta$ changes the sign from a negative value for dendritic morphologies to a positive value for globular shapes. The transitional value $\delta=0$ corresponds to the composition $\mathrm{Ni}_{60} \mathrm{Cu}_{20} \mathrm{Cr}_{20}$ and is hence in good agreement with the transition point given by the vanishing diagonal part of the isoline. The transition takes place in a smooth manner. On the one hand, this follows from the linear increasing of the growth velocity $V$ of the dendritic or globular tip with increasing $\mathrm{Cr}$ concentration (see the values for $V$ in Fig. 1). On the other hand, the smooth transition is confirmed by the smooth dependence of the deviation $\delta$ on the melt composition, Fig. 2. The morphology of the growing structure is determined by the concurrence of the stabilizing force due to the surface energy and of the destabilizing force due to the concentration gradients on the interface [1,2]. The morphological transition can be explained considering the balance between the stabilizing and destabilizing forces. During the computed transition from a dendritic to globular shape for varying composition of the ternary alloy $\mathrm{Ni}_{60} \mathrm{Cu}_{40-x} \mathrm{Cr}_{x}$ with $0<x<40$, the liquidus slope and the deviation of the equilibrium partition coefficient from unity decrease.
This leads to a reduction of the destabilizing forces on the interface. The globular growth shapes are becoming stable against perturbations. Although the phase-field simulations of the ternary system were conducted for fixed undercooling, we remark that a similar morphological transition from dendritic to globular growth shapes has been observed in numerical simulations of dendritic growth in binary alloys [10], where the initial undercooling acts as a control parameter for the transition. In this case a morphological transition occurs through a coalescence of side branches with increasing of the undercooling.

In summary, our numerical simulations show the occurrence of a morphology transition from dendritic to globular growth structures in the ternary $\mathrm{Ni}-\mathrm{Cu}-\mathrm{Cr}$ system for a varying alloy composition. The results demonstrate the general potential of the phase-field approach in modeling multicomponent solidification in a thermodynamically consistent way. Together with previous developments [5], the work in this Letter shows that the diffuse interface formulation provides a uniform description for modeling complex microstructure evolution in pure, binary, and multicomponent alloy systems.

We thank P. K. Galenko from DLR Cologne for helpful discussions and gratefully acknowledge the financial support provided by the German Research Foundation (DFG) under Grants No. Ne 882/2-1 and No. Ne 882/2-2.

[1] W.W. Mullins and R. F. Sekerka, J. Appl. Phys. 35, 444 (1964).

[2] R. Trivedi and W. Kurz, Acta Metall. 34, 1663 (1986).

[3] S. R. Coriell, G. B. McFadden, and P.W. Voorhees, J. Cryst. Growth 82, 295 (1987).

[4] G. Caginalp and W. Xie, Phys. Rev. E 48, 1897 (1993); K. R. Elder et al., Phys. Rev. E 64, 021604 (2001); D. Kessler, J. Cryst. Growth 224, 175 (2001); C. Eck, Multiscale Model. Sim. 3, 28 (2004).

[5] W. J. Boettinger, J. A. Warren, C. Beckermann, and A. Karma, Annu. Rev. Mater. Res. 32, 163 (2002).

[6] J. A. Warren and W. J. Boettinger, Acta Metall. Mater. 43, 689 (1995).

[7] J. J. Hoyt, M. Asta, and A. Karma, Phys. Rev. Lett. 86, 5530 (2001); J. J. Hoyt, B. Sadigh, M. Asta, and S. M. Foiles, Acta Mater. 47, 3181 (1999); D. Y. Sun, M. Asta, and J. J. Hoyt, Phys. Rev. B 69, 024108 (2004).

[8] H. Garcke, B. Nestler, and B. Stinner, SIAM J. Appl. Math. 64, 775 (2004).

[9] N. Provatas et al., Phys. Rev. Lett. 80, 3308 (1998); A. Karma and W.-J. Rappel, Phys. Rev. E 57, 4323 (1998); M. Plapp and A. Karma, Phys. Rev. Lett. 84, 1740 (2000); W. L. George and J. A. Warren, J. Comput. Phys. 177, 264 (2002).

[10] P. K. Galenko and V. A. Zhuravlev, Physics of Dendrites: Computational Experiments (World Scientific, Singpore, 1994). 